\title{
CAPACITAÇÃO PARA A PRÁTICA BASEADA EM EVIDÊNCIAS: RELATO DE EXPERIÊNCIA
}

\section{Training for evidence-based practice: experience report}

\author{
Bruna Dedavid da Rocha \\ Centro Universitário Franciscano - UNIFRA - Santa Maria - (RS) Brasil \\ Cláudia Zamberlan \\ Centro Universitário Franciscano - UNIFRA - Santa Maria - (RS) Brasil \\ Dirce Stein Backes \\ Centro Universitário Franciscano - UNIFRA - Santa Maria - (RS) Brasil
}

\section{RESUMO}

Objetivo: Relatar a experiência de uma capacitação para residentes em enfermagem obstétrica, acerca de temas relevantes na área, por meio da prática baseada em evidências. Síntese dos Dados: Os dados podem ser sintetizados do seguinte modo: trata-se de um trabalho oriundo de atividade desenvolvida na disciplina de Tecnologias Inovadoras e Empreendedorismo em Enfermagem e Saúde, do Mestrado Profissional em Saúde Materno Infantil. A tecnologia de cuidado que emergiu foi uma capacitação para residentes em enfermagem obstétrica de uma instituição de ensino superior. O trabalho foi realizado no segundo semestre de 2016 e seguiu os passos elencados por um diagrama teórico da disciplina, baseado na pesquisa-ação. Importa acentuar que foi realizada a capacitação na disciplina de Seminários Integrados, com residentes em enfermagem obstétrica, a partir da percepção das mesmas, de dificuldades na implementação da prática baseada em evidências em seu cotidiano. A capacitação pode ser considerada uma tecnologia de cuidado, visto que, possibilita a educação em saúde das pós-graduandas e instiga a realização de pesquisas bem delineadas na área obstétrica. Conclusão: Acentua-se existir grande necessidade de valorização da profissão de enfermeiras obstetras nas maternidades e de se dever explorar mais sua importância na mudança do modelo obstétrico assistencial brasileiro.

Descritores: Enfermagem Obstétrica; Prática Clínica Baseada em Evidências; Tomada de Decisões.

\section{ABSTRACT}

Objective: To report the experience of a training for residents in obstetrical nursing, on relevant topics in the area, through evidence-based practice. Data synthesis: The data can be summarized as follows: this is a work from an activity developed in the discipline of Innovative Technologies and Entrepreneurship in Nursing and Health, of the Professional Masters in Maternal and Child Health. The care technology that emerged was a training for residents in obstetric nursing of a higher education institution. The work was carried out in the second half of 2016 and followed the steps listed by a theoretical diagram of the discipline, based on action research. We can say that a training was provided in the discipline of Integrated Seminars, with residents in obstetric nursing, from their perception of difficulties in implementing the practice based on evidence in their daily life. Training can be considered a technology of care, since it enables the postgraduate health education and instigates well-designed obstetrical research. Conclusion: After all, there is a great need to value the obstetrical nurse profession in maternity hospitals and its importance in changing the Brazilian obstetric care model.

Descriptors: Obstetric Nursing; Evidence-Based Practice; Decision Making. 


\section{INTRODUÇÃO}

A prática baseada em evidências científicas consiste em utilizar os melhores resultados disponíveis, oriundos de estudos metodologicamente consistentes, da literatura nacional e internacional, para balizar a decisão clínica na assistência à saúde. No Brasil, o movimento da prática baseada em evidências está melhor alicerçado na medicina, sendo que na área da enfermagem ainda se encontra em fase de ascensão ${ }^{(1)}$.

A utilização de evidências científicas, na área obstétrica e perinatal, vêm se destacando no Brasil e no mundo, pela necessidade de modificar o paradigma da assistência ao parto, o qual ainda se utiliza principalmente da experiência prévia dos profissionais e de livros que não atualizados. Existem inúmeras movimentações, principalmente por parte da enfermagem obstétrica, para que, as melhores evidências científicas sejam utilizadas na prestação do cuidado à mulher e ao neonato( ${ }^{(2)}$.

Por meio dos resultados encontrados na pesquisa Nascer no Brasil, ainda existe elevado índice de intervenções desnecessárias durante o trabalho de parto e o parto, como a episiotomia e a manobra de kristeller, as quais não possuem evidências de benefícios para sua utilização. A partir dessas constatações, manifesta-se a relevância da mudança do modelo obstétrico brasileiro e a necessidade de estratégias para a implementação das práticas baseadas em evidências, na assistência ao nascimento, de maneira a qualificar e humanizar o cuidado ${ }^{(3)}$.

Porém, para a implementação da prática baseada em evidências, ainda existem desafios a serem superados, como a questão de suporte organizacional, autonomia do enfermeiro para prestar essa assistência mais qualificada e a educação permanente como ferramenta de motivação e mudança de atitude dos profissionais ${ }^{(4)}$.

Nessa perspectiva, justifica-se a necessidade de uma capacitação para residentes em enfermagem obstétrica, pela percepção de que a prática baseada em evidências deve fazer parte do cotidiano do enfermeiro obstetra. Isso se deve principalmente, pelo momento atual da área, a qual está passando por um processo de modificações de contexto. Consequentemente, também se destaca a ascensão e a valorização desse profissional, pela sua percepção ampliada de cuidado, compreensão e respeito ao processo fisiológico do trabalho de parto e do parto. Além dessas condições, é de extrema importância a qualificação profissional para a pesquisa, visto que a mesma é uma grande aliada na implementação das boas práticas de atenção ao parto, ao nascimento e a instrumentalização dos profissionais e dos serviços de saúde.

Diante do exposto, nessa base de pensamento, este estudo tem por objetivo relatar a experiência de uma capacitação para residentes em enfermagem obstétrica, acerca de temas relevantes na área, por meio da prática baseada em evidencias, como uma tecnologia de cuidado em saúde.

\section{SÍNTESE DOS DADOS}

O presente trabalho foi elaborado na disciplina de "Tecnologias Inovadoras e Empreendedorismo em Enfermagem e Saúde", do Programa de Pós-graduação em Saúde Materno Infantil do Centro Universitário Franciscano, Santa Maria, RS, Brasil.

Importa descrever a experiência da capacitação para residentes como tecnologia de cuidado. Configura-se esse procedimento como uma atividade desenvolvida na disciplina de Tecnologias Inovadoras e Empreendedorismo em Enfermagem e Saúde, do Programa de Mestrado Profissional em Saúde Materno Infantil. A tecnologia de cuidado que emergiu foi conceituada como processo e direcionada para a capacitação de residentes em enfermagem obstétrica de uma instituição de ensino superior. O trabalho foi realizado no segundo semestre de 2016 e seguiu os passos elencados por um diagrama teórico da disciplina, que se apresentam em seguida: identificação do problema, análise justificada do problema com a equipe envolvida, pesquisa na literatura nacional e internacional, discussão de estratégias coletivas para a resolução do problema, implementação do processo/tecnologia, avaliação geral do processo/tecnologia e elaboração do relatório final do processo.

O diagrama teórico segue os passos preconizados pela metodologia da pesquisa-ação, sendo que esse modelo qualitativo consiste em um tipo de pesquisa de base social e empírica, que se alia com a ação, para encontrar soluções que envolvem problemas levantados pelo pesquisador e/ou pelos participantes, os quais envolvem-se de forma mútua, em todo o processo ${ }^{(5,6)}$.

A pesquisa-ação engloba etapas que permeiam a identificação de um problema de pesquisa em âmbito social e/ ou institucional, o levantamento de dados pelos participantes e a análise e identificação dos significados encontrados. Além dessas fases do processo, envolve a intervenção na prática, objetivando provocar transformações em um determinado contexto(7). 
Neste estudo, serão utilizadas ações estratégicas, as quais se fundamentam no planeamento prévio de uma transformação, somente pelo pesquisador, sem a participação direta dos participantes envolvidos no processo. Nesta classificação, apenas o pesquisar irá acompanhar e avaliar os resultados encontrados da aplicação da tecnologia de transformação( ${ }^{(7)}$.

As etapas do diagrama metodológico foram discutidas com base em autores destacados pelo seu envolvimento na área materno infantil, inter-relacionados aos princípios do empreendedorismo e inovação em enfermagem/saúde.

Passa-se a expor a forma de se aliar a pesquisa-ação com a prática baseada em evidências na área obstétrica.

Por meio da disciplina de Seminários Integrados, ofertada para pós-graduandas do Programa de Residência em Enfermagem Obstétrica, de uma instituição de ensino superior, foi levantada a necessidade de trabalhar a prática baseada em evidências, a partir de temáticas emergentes na área. Essa observação se deve à experiência prévia da mestranda que realiza esse estudo, em um curso sobre "Parteria Baseada em Evidências", que ocorreu no mês de outubro de 2016, em São Paulo, promovido pela "Levatrice - cursos para parteiras".

Também foi identificado ser útil esse trabalho, em uma participação da pesquisadora na disciplina de Seminários Integrados, partindo de algumas dúvidas e dificuldades emergentes das residentes, diante da prática obstétrica e do conhecimento das melhores e mais atuais evidências científicas, a serem aplicadas na prática clínica.

Nesse enfoque, a liderança do enfermeiro pode ser expressa por meio das pesquisas desenvolvidas pelos profissionais, no estabelecimento de políticas públicas, estratégias, gestão da rede de atenção, o que proporciona o acesso da população aos serviços de saúde, por meio de uma cobertura universal. $\mathrm{Na}$ área da enfermagem obstétrica, justifica-se a imprescindível articulação das ações, da rede de atenção e o desenvolvimento de parceiras, as quais podem ser oportunizadas por uma liderança consistente na enfermagem. Além dessa consideração, a pesquisa também possui um papel relevante para demostrar os impactos das ações desenvolvidas pela enfermagem e para se alcançar o acesso universal da assistência à saúde, o que consequentemente resultará em uma maior valorização e reconhecimento da profissão(8).

Acresce esclarecer que, a partir de uma demanda nacional pela mudança de práticas e do modelo de assistência obstétrica, as políticas públicas iniciaram um processo de valorização e instrumentalização de enfermeiras obstetras, para atuarem nesse novo contexto de saúde materno-infantil. Nessa vertente emergente, estratégias tais como a Rede Cegonha, oportunizaram a abertura de editais para especializações e residências em enfermagem obstétrica, em diferentes regiões do país. Todavia, a fim de promover essas modificações, tão necessárias em um contexto em que a cesariana é a principal via de parto, as intervenções obstétricas são realizadas rotineiramente e as pacientes sofrem violência obstétrica, o que caracteriza uma violação dos direitos das mulheres ${ }^{(9)}$.

Importa acentuar que, desde 1996, a Organização Mundial da Saúde (OMS) preconiza e incentiva boas práticas de atenção ao parto e ao nascimento, baseadas nos melhores estudos disponíveis, principalmente em metanálises, disponíveis em Cochrane Collaboration. Nesse domínio de informação, trata-se de ter acesso a um guia sobre as práticas que devem ser estimuladas ou não, durante o processo parturitivo( ${ }^{(10)}$. Em estudo publicado em 2010, foi elaborada uma reflexão crítica após 10 anos da implementação do referido guia da OMS. Os autores concluíram que a não adesão dos profissionais às "melhores práticas clínicas" na assistência se deve principalmente à falta de conhecimento sobre o organismo feminino, ou seja, ao desconhecimento dos profissionais sobre as necessidades da mulher e do processo fisiológico envolvido no parto e no nascimento. Importa pois conhecer o que o parto provoca nesse grupo em estudo(11).

A partir dessas evidências, as práticas menos invasivas poderão sem implementadas. Essa atenção implica haver ainda o desconhecimento do processo fisiológico e da compreensão ampliada do ser humano, entendido como integral e único. Dessa questão decorre que se acaba por repercutir o trabalho de parto e o parto em um maior uso das tecnologias, nomeadamente pelo receio de desfechos negativos. Porém, quanto mais intervenções são realizadas em um processo natural, como no caso do parto de risco habitual, maiores são as chances de complicações obstétricas ${ }^{(2)}$.

No entanto, importa dizer que a partir das Diretrizes para a Assistência ao Parto Normal, documento ministerial lançado em 2017, se busca reduzir as taxas de intervenções desnecessárias no trabalho de parto e no parto, tais como as seguintes práticas correntes: episiotomia de rotina, indução do parto com ocitocina e elevada taxas de cesarianas, as quais muitas vezes são mal indicadas pelos profissionais médicos ${ }^{(12)}$. Para além de nortear a prática dos profissionais de saúde, as referidas Diretrizes basearam-se também em evidências científicas. Também se preconiza empoderar as mulheres para que as mesmas possam tomar uma decisão informada em relação à assistência que desejam, na assistência ao parto e nascimento ${ }^{(12)}$. 
Portanto, percebe-se que existe uma grande movimentação no âmbito das políticas públicas de saúde, para qualificar a assistência ao parto e ao nascimento, baseada nos estudos mais recentes. A partir dessa constatação, a implementação de boas práticas apresenta aporte teórico consistente, para serem usadas pelos profissionais nas instituições de saúde.

Teve reconhecido mérito, entretanto, um evento internacional, denominado de Simpósio Internacional de Assistência ao Parto (SIAPARTO). Essa iniciativa ocorre anualmente no Brasil e nela se discutem as atualizações de evidências científicas, na área obstétrica. Deslocam-se ao Simpósio especialistas e pesquisadores de diversos países. Pode afirmar-se que, em 2015, o professor de obstetrícia e perinatologia Frank Lowen, da Alemanha, colaborou com o evento. Nele realizou uma palestra em que debateu a fisiologia do trabalho de parto (prostagladinas versus ocitocina). Referiu ainda os parâmetros contemporâneos de avaliação da progressão, como o uso de partograma de Zahng, sendo o partograma um instrumento obrigatório nas maternidades, para o acompanhamento do trabalho de parto e do parto. Porém o gráfico utilizado em muitos serviços nem considera as individualidades do processo, nem os parâmetros de dilatação e de descida fetal, preconizados pelos últimos estudos. Nessa circunstância acaba por se darem intervenções desnecessárias e que podem causar riscos maternos e infantis, tais como a amniotomia de rotina, a indução com ocitocina e a cesariana. Essas práticas, se empregadas sem necessidade, podem ter repercussões negativas na vitalidade fetal. Referiu-se um dos importantes temas debatidos nesse Simpósio, realizado no Brasil.

Nessa vertente de discussão, foi realizada no dia 7 de novembro de 2016, uma capacitação para as residentes, intitulada "Parteria Baseada em Evidências: novas perspectivas", na instituição de ensino superior suprarreferida, sendo realçados temas emergentes da prática obstétrica, tais como os seguintes: fisiologia do parto normal, ausculta intermitente dos batimentos cardiofetais e cardiotocografia, avaliação da progressão do trabalho de parto por parâmetros contemporâneos (partograma de Zahng), métodos de avaliação da progressão sem toques, manejo do terceiro estágio do trabalho de parto, períneo e, em atenção, ao parto e ao parto na água. Estes temas não foram trabalhados no seu todo, embora fossem focados pontos específicos, trazendo os estudos e consensos nacionais e internacionais mais recentes sobre determinadas condutas.

Inicialmente, o processo fundamentou-se no seguinte questionamento prévio sobre a capacitação: "quais são as dúvidas mais prevalentes que vocês - residentes - apresentam em relação à prática obstétrica?" As residentes pontuaram o manejo no período expulsivo do trabalho de parto como a principal dificuldade, principalmente pelo receio de intercorrências obstétricas que podem ocorrer nesse momento. Também ressaltaram a ausência de um protocolo ou de um consenso entre todos os profissionais que atuam na maternidade, sobre as condutas a serem tomadas. Essa falta de consenso acaba por dificultar a implementação de uma assistência qualificada e humanizada nas orientações dirigidas às mulheres e seus familiares. Acresce acentuar que as residentes enfatizaram que cada profissional, principalmente o/a médico/a, acaba tendo uma conduta individual, na maioria das vezes não centrada na necessidade da parturiente ou nas melhores evidências científicas. Nessa posição, o/a profissional baseia-se no que acredita ser "o melhor e o mais seguro, naquele momento".

Essa proposta de capacitação vem ao encontro de um cuidado de enfermagem empreendedor, na medida em que proporciona estímulo para que as pós-graduandas repensem as suas práticas na obstetrícia, de maneira a implementarem ações para a mudança de realidades locais, que pode reverberar em uma mudança maior no modelo obstétrico no Estado e no Brasil. Essa proposta também se justifica, a atender a que permite que se repense a articulação de redes de atenção e parcerias, bem como o empoderamento das usuárias, por iniciativas de educação em saúde, no intuito de possibilitar transformações sociais, a cidadania e a maior justiça social(13).

$\mathrm{Na}$ área da enfermagem, o mercado de trabalho está exigindo cada vez mais que o profissional tenha uma postura de liderança, empreendedorismo e proatividade. Essas orientações sociais significam que se proponham processos de atualização ao longo da vida, em prol de uma assistência qualificada, para que se possa oferecer aos pacientes a melhor prática de cuidado. Para tanto, o profissional precisa aventurar-se em novas ideias e perspectivas de trabalho, por meio de um olhar crítico e reflexivo de sua intervenção informada. A partir dessa constatação, a proatividade torna-se uma qualidade essencial, a favor de uma ação que contribui para as mudanças no ambiente de trabalho(14).

Portanto, uma das estratégias que pode emergir da capacitação das residentes será a criação de protocolos e consensos acerca dos temas elencados, com o objetivo de introduzir um discurso informado, debatido e crítico dos profissionais. Além dessa mudança, partindo do princípio de que poderão surgir novos estudos e as práticas podem se modificar constantemente, a capacitação profissional implica um processo contínuo de formação, a ser implementada nos serviços de saúde. Por consequência, se possibilita que as mulheres gestantes tenham acesso a uma assistência mais adequada e mais qualificada, conforme é preconizado pelas políticas públicas de saúde, como a Política de Humanização do Parto e Nascimento (PHPN) e a Rede Cegonha. 
Em relação à avaliação do desenvolvimento do trabalho, a mestranda pode entender em profundidade o interesse das residentes em aprimorar seus conhecimentos na área obstétrica. Foi visualizado que existem ainda algumas lacunas relacionadas à fisiologia do trabalho de parto e ao parto, Podem ser nomeadas as seguintes limitações: a compreensão de respeitar cada período desse processo e repassar essa circunstância para que a equipe de saúde também tenha essa postura observada. Ademais, as residentes também demonstraram interesse a respeito da temática de ultrassonografia obstétrica de risco habitual, importante principalmente na assistência pré-natal, o que será desenvolvido em uma outra oportunidade, durante a disciplina de Seminários.

Portanto, a formação foi considerada uma ação inovadora e empreendedora, pois tem um alcance bem além da capacitação no momento. Sendo uma ação ampla, objetiva mudanças de práticas no cotidiano de trabalho, por meio de um processo constante de construção e desconstrução de conhecimento. Nesse modo de trabalho informado por evidências se instiga e elaboração de novas metodologias de intervenção nos cenários em que os profissionais atuam, com vistas à conquistar a transformação social(15).

A realização de pesquisas baseadas em evidências justifica-se junto de residentes, visto que, possui um elevado valor de transformação das práticas obstétricas, principalmente no que se refere à redução das intervenções desnecessárias, com a possibilidade de introduzir novas tecnologias de cuidado, que respeitem o processo de parto e de nascimento fisiológico, dito que as mulheres venham a ter experiências positivas.

\section{CONCLUSÃO}

Diante do exposto, argumenta-se que a capacitação para a prática baseada em evidências pode ser considerada uma tecnologia empreendedora na saúde materno infantil. Afinal, as evidências científicas devem nortear a prática clínica, se bem que nem todos os profissionais acedam aos melhores estudos publicados, o que impossibilita que a tomada de decisão clinica seja pautada no melhor cuidado a ser ofertado. Possibilitar acesso às melhores referências na área também é uma maneira de instigar os profissionais, em particular, as residentes, a atualizarem-se e a produzirem pesquisas metodologicamente rigorosas na área. A pesquisa é de extrema relevância, principalmente para a classe de enfermagem obstétrica, na medida em que seja uma categoria profissional de valorizar e cujo conhecimento seja reconhecido.

\section{REFERÊNCIAS}

1. Galvão CM, Sawada NO, Mndes IAC. A busca das melhores evidências. Rev Esc Enferm USP. 2003; 37(4):4350, 2003.

2. Ministério da Saúde(BR). Cadernos HumanizaSUS. Humanização do parto e nascimento. Ministério da Saúde. Universidade Federal do Ceará. Brasília (DF):2014.

3. Leal MC, Pereira APE, Domingues RMSM, Filha MMT, Dias MAB, Pereira MN; et al. Intervenções obstétricas durante o trabalho de parto e parto em mulheres brasileiras de risco habitual. Cad Saúde Pública. 2014; 30 (sup): $17-47$.

4. Côrtes CTC, Santos RCS, Caroci AS, Oliveira SG, Oliveira SMJV, Riesco MLG. Metodologias de implementação de práticas baseadas em evidências científicas na assistência ao parto normal: estudo piloto. Rev Esc Enferm USP.2015; 49(5):716-25.

5. Thiollent M. Metodologia da pesquisa-ação. São Paulo: Cortez; 1986.

6. Thiollent M. Pesquisa-ação nas organizações. São Paulo: Atlas; 1997.

7. Koerich MG, Backes DS, Sousa FGM, Erdmann AL, Albuquerque GL. Pesquisa-ação: ferramenta metodológica para a pesquisa qualitativa. Rev Eletrônica Enferm. 2009;11(3): 717-23.

8. Mendes IAC, Ventura CAA, Trevizan MA, Marchi-Alves LM, Souza-Junior VD. Educação, liderança e parcerias: potencialidades da enfermagem para a cobertura universal de saúde. Rev Latinoam Enferm. 2016;24: e2673.

9. Camacho KG, Progianti JM. A transformação da prática obstétrica das enfermeiras na assistência ao parto humanizado. Rev Eletrônica Enferm.2013; 15(3):648-55.

10. Cochrane Brasil. Disponível em: https://brazil.cochrane.org/search/site/guias\%20partos 
11. Rocha JA, Novaes PB. Uma reflexão após 23 anos das recomendações da Organização Mundial da Saúde para parto normal. Femina. 2010;38(3):120-6.

12. Ministério da Saúde (BR). Diretriz Nacional de Assistência ao Parto Normal. Brasília(DF); 2017.

13. Backes DS, Erdmann AL, Buscher A. O cuidado de enfermagem como prática empreendedora: oportunidades e possibilidades. Acta Paul Enferm.2010; 23(3):341-7.

14. Ferreira GE, Dall'Agnol CM, Porto AR. Repercussões da proatividade no gerenciamento do cuidado: percepções de enfermeiros. Esc Anna Nery Rev Enferm. 2016;20(3):e20160057.

15. Backes DS, Erdmann AL. Formação do enfermeiro pelo olhar do empreendedorismo social. Rev Gaúch Enferm. 2009; 30(2): 242-8.

Endereço para correspondência:

Bruna Dedavid da Rocha

Centro Universitário Franciscano - UNIFRA

Rua dos Andradas, 1614

Bairro: Centro

CEP: 97010-032 - Santa Maria - RS - Brasil

E-mail: brunadedavid.rochaa@gmail.com 